\section{Linguistic and imaginal mnemonics in paired-associate recall*}

\author{
EDWIN G. AIKEN \\ Naval Personnel and Training Research Laboratory, San Diego, Calif. 92152
}

A comparison was made between mental imagery and sentence memorization as mnemonic aids to the recall of noun-noun paired associates. Data were obtained to allow postexperimental selection of Ss who appeared to have been most proficient at carrying out the instructional sets associated with each mnemonic. Data from these Ss were analyzed within a design permitting a study of noun similarity and associational direction. Mental imagery was superior to sentence memorization, recall of nonsimilar nouns superior to similar, and forward recall superior to backward. Also, a significant interaction indicated that noun similarity produced relatively greater difficulty for the image-mnemonic Ss. It is concluded that the use of mental imagery in recall cannot be reduced entirely to linguistic mechanisms.

Considerable research interest has recently centered on the utility of mental imagery as a theoretical construct in verbal learning and recall (Bower, 1969; Bugelski, 1970; Paivio, 1969). However, the private nature of mental images makes it difficult not only to define the mechanisms of their effects, but to have assurance that the Ss are even engaging in imaginal activity. These problems, combined with certain epistomological biases, have predisposed radical behaviorists to equate, or parallel, mentalistic constructs such as images with some sort of linguistic activity. An important theoretical question, therefore, is whether or not such equating is justified. Can mental images be shown to aid recall independent of the mnemonic value of any hypothetical linguistic correlates?

Bower (1969) reported a number of studies which circumstantially implicate images as independent aids to recall. His research and that of others (Paivio \& Yuille, 1969; Paivio \& Foth, 1970; Rimm, Alexander, \& Eiles, 1969) has attempted to compare mnemonic strategies through different instructions given the Ss. A question always remains as to whether or to what degree the $S s$ are, in fact, following the instructions. If, as is likely, Ss vary in their ability to form mental images, then those low in the ability might seek out a linguistic mnemonic despite instructions. Similarly, Ss particularly adept at imagery might use this aid even if explicitly istructed to use another mediator.

Paivio \& Yuille (1969) asked Ss zbout the strategy they employed luring learning and found that an

\footnotetext{
* The opinions and assertions contained lerein are those of the writer and are not to se construed as official or as reflecting the riews of the Navy Department or naval iervice.
}

imaginal strategy was preferred and most effective with concrete nouns. Paivio \& Foth (1970) had Ss instructed to mediate imaginally to draw their images, and those instructed to mediate verbally to write their sentences. Under these conditions, verbal mediation was superior with abstract nouns and imaginal instructions were superior with concrete nouns.

The goal of the research here reported was to compare a linguistic and an imaginal mnemonic aid to PA learning and recall within a design which provided independent evidence on the extent to which Ss given different instructions were, in fact, doing something different, so that only Ss apparently most successful with each strategy could be compared. SUBJECTS

The Ss were Navy recruits divided

$$
\text { LEARNING }
$$

The basic task involved memorizing 32 noun pairs, each of which had been imbedded in a sentence. The nouns were selected from the list scaled by Paivio, Yuille, \& Madigan (1968). Only equally into two groups of 22 . nouns with imagery (I) ratings of 6 or greater on a scale of 7, together with very high Thorndike-Lorge frequency of occurrence ratings, were used. An attempt was also made to keep their meaningfulness $(m)$ and concreteness (c) ratings as nearly the same as possible. To provide data on any interaction between associational confusion and type of mnemonic aid, 16 of the 64 nouns were selected to make up eight meaningfully similar sets of 2 each, e.g., flower and blossom, sea and ocean, college and university. The remaining 48 nouns, on the other hand, were selected so as to have no direct meaningful similarity among themselves or with the meaningfully similar nouns. Each of the meaningfully similar nouns was imbedded in a different sentence. Thus, 16 of the 32 sentences contained a noun which was meaningfully similar to one of the nouns in another sentence, while the remaining 16 sentences contained nouns not meaningfully similar to any of the nouns imbedded in any of the 32 sentences.

Certain rules governed the pairing of nouns. First, pairings with very high probability associations, e.g., baby-crib, were avoided. Second, one of the nouns with a meaningfully similar counterpart appeared as the first and the other as the second noun in their respective sentences. Table 1 presents the 32 noun pairs. The first eight pairs in each column have the meaningfully similar nouns underlined. The order of the 32 sentences was determined randomly.

Each sentence was constructed such that the two nouns in each pair occurred in the orders shown in Table 1. Sentences were written in which the imbedded nouns were in some sort of interaction. The sentences were presented to the Ss in booklets, with one sentence per page. The nouns to be associated were underlined and capitalized in each

Table 1

Noun Pairs Imbedded in Sentences for Memorization

$\begin{array}{ll}\text { 1. Hall-Car* } & \text { 2. Automobile-Coffee } \\ \text { 3. Bottle-Flower } & \text { 4. Blossom-Corner } \\ \text { 5. Winter-House } & \text { 6. Home-Arm } \\ \text { 7. Sea-Fire } & \text { 8. Truck-Ocean } \\ \text { 9. Railroad-University } & \text { 10. College-Palace } \\ \text { 11. Rock-Toy } & 12 \text {. Horse-Stone } \\ \text { 13. Baby-Shore } & 14 \text {. Coin-Infant } \\ \text { 15. Mother-Student } & 16 . \text { Pupil-Girl } \\ \text { 17. Wine-Judge } & 18 . \text { Circle-Building } \\ \text { 19. Mountain-Cottage } & 20 . \text { Bird-Meat } \\ \text { 21. Cell-Nail } & \text { 22. Book-Frog } \\ \text { 23. Cabin-Door } & 24 . \text { Church-Grass } \\ \text { 25. Table-Engine } & 26 . \text { Grandmother-Chair } \\ \text { 27. Policeman-Tower } & \text { 28. Street-Queen } \\ \text { 29. Sugar-Ship } & \text { 30. Furniture-Pipe } \\ \text { 31. Village-Camp } & \text { 32. Cat-Window }\end{array}$

*Each of the first eight pairs in the first column contains a meaningfully similar noun directly opposite in the second column. They are underlined. 
sentence. Example sentences are: "The HALL echoed from the sounds of the little boy racing his tiny CAR," and "The angry MOTHER was yelling at the demonstrating STUDENT." LEARNING:

\section{INSTRUCTIONAL SETS}

Half the Ss were given instructions to use a mental-imagery strategy to aid associating the noun pairs, while the other half were instructed to memorize the sentences as an aid. All Ss were given $10 \mathrm{sec}$ to study each sentence and told that the recall test at the end would require that they respond with one of the underlined-capitalized words from each sentence when shown the other. Ss instructed in an imaginal mnemonic were told to create as vivid and active a mental image as possible of the scene described in each sentence to be used later as a context for recall of the noun pairs. Ss instructed to learn the sentences were told to try to memorize exactly each sentence to be used later as a context for associating the noun pairs.

Both to increase the probability that the instructional sets were being followed and to index roughly individual differences in the ability to follow them, $S$ in both instructional groups were required to carry out two different subsidiary tasks parallel to the primary learning. In the mental-imagery-instructed group, Ss turned the pages in their booklets following the 10-sec study period on each sentence and found two questions asking for more detailed elaboration of their images. For example, to the sentence presented above involving the noun pair, Hall-Car, the questions were: "Is the hall carpeted?" and "What color is the tiny car?" The two questions were followed by a place to record, on a 5 -point scale, a rating of the intensity of the image aroused by the sentence. Before proceeding to the next sentence, 230 sec were allowed for answering the two questions and making the rating. For the Ss instructed to memorize the sentences, the subsidiary task following each 10 -sec study period involved correcting a modified version of the sentence they had just studied until it read exactly like the original. The modifications involved adding, deleting, or substituting words. To avoid possible unlearning, none of the sentence modifications involved the noun pairs to be associated, and they were again underlined. Thirty seconds was also allowed for this task.

\section{RECALL}

Immediately following study of the 32 sentences and again $2 \frac{1}{2} \mathrm{~h}$ later, a test for recall of the noun pairs was carried out. Each $\mathrm{S}$ was given a sheet of paper with 32 nouns and asked to write the noun that went with each in the 32 sentences. Half of the nouns on the sheet had appeared as the first noun and half as the second in the sentences, i.e., half required $S$ to produce a forward and half a backward association. In the test, half of the meaningfully similar nouns, i.e., nouns meaningfully similar to nouns in other sentences, required forward and half backward association. A constraint was that the meaningfully similar nouns were always to be written in by $S$; they never appeared as the cueing nouns on the recall sheet. Similarly, half of the nouns from sentences containing meaningfully dissimilar nouns required forward and half backward association. The order of the nouns was determined randomly for the two testing occasions, with the constraint that at least three items intervene between nouns requiring responses from the meaningfully similar noun set. For example, the noun requiring the word "baby" as the correct response was at least three nouns separated from the noun calling for "infant" as the response. Ss were given $10 \mathrm{~min}$ to write down as many noun associates as possible.

Finally, a second form of the sentence modification-correction task was administered to both instructional groups. This was done to permit comparison of the memorization of the group instructed to memorize with the incidental memorization of the group instructed to form mental images. Such data were to be related to the hypothesis that imagery-instructed Ss also store verbal codes (Bower \& Winzenz, 1970) and to determine the degree to which their recall scores could as well be attributed to verbal memorization as to imagery mediation.

\section{RESULTS AND DISCUSSION}

The first step in the analysis involved examining the data from the tasks designed to select those Ss who were best carrying out the mnemonic strategy given them in their instructions. These were the sentence modification-correction task for the sentence memorization group and the rates image intensity for the mental imagery group.

For the sentence memorization group, a random sample of eight of the modified sentences was chosen and scored for each $\mathrm{S}$. A point was given for each modification detected and correctly changed and a point subtracted for each modification missed or incorrectly changed. The total points over the eight sentences was taken to represent the accuracy with which $S$ had been able to carry out the instructions to memorize exactly the sentences. Because the delayed test for $P A$ recall was considered to be a more stringent test of the two mnemonic strategies than the immediate test, the delayed sentence modification-correction scores were used for selecting Ss.

For the mental imagery group, a rated image intensity score was obtained for each $S$ by summing the ratings over the 32 sentences. For subsequent analyses, the data on the highest and lowest scoring half of each instructional group was compared separately ( $\mathrm{N}=11$ in each group).

Obviously, this selection of Ss did not rule out the possibility that the instructional sets were still being violated, but it did provide subsets of Ss for both groups who appeared to be best at the particular mnemonic aid assigned them. Some additional e vidence on what the imagery-instructed Ss were doing was obtained $\mathrm{fm}$ the extent of their incidental sentence memorizing as compared with the intentional memorizing of the other group on the delayed sentence modificationcorrection task. This was examined, and although some incidental memorization of the sentences was shown, it was significantly less $(t=4.04, p<.005)$ than that shown by the $S s$ instructed to memorize. Also, within the imagery-instructed group, the correlation between incidental sentence memorization and PA recall was .03 , implying little, if any, reliance on verbal storage by imagery-instructed Ss. Although no similar check on incidental imaginal activity by the sentence-memorization Ss was obtained, if such had been taking place it would be an artifact working against the experimental hypothesis of an independent contribution of mental imagery to recall.

Having selected the subsets of Ss from each mnemonic condition, an analysis of variance was next performed on the delayed $\mathrm{PA}$ recall scores of the subsets of Ss assumed to be most closely following the instructions given them. In addition to the between-S variable of instructional set, the analysis contained two factorially arranged within-S sources of variance, i.e., whether a meaningfully similar or dissimilar noun was to be recalled and whether recall was backward or forward. The results indicated superior recall for the group instructed to employ a mental imagery mnemonic $(F=8.19, p<.01)$, for the recall of meaningfully dissimilar nouns $(F=15.27, p<.001)$, and for forward recall $(F=5.61, p<.05)$. In addition, the Instructional Set by Meaningfully Similar-Dissimilar Noun interaction was significant $(F=25.39, p<.001)$. 
Table 2

Proportion of Correctly Recalled Paired Associates

\begin{tabular}{|c|c|c|c|c|}
\hline & \multicolumn{2}{|c|}{ Imaginal Instructions } & \multicolumn{2}{|c|}{ Memorization Instructions } \\
\hline & $\begin{array}{c}\text { Forward } \\
\text { Recall }\end{array}$ & $\begin{array}{l}\text { Backward } \\
\text { Recall }\end{array}$ & $\begin{array}{l}\text { Forward } \\
\text { Recall }\end{array}$ & $\begin{array}{l}\text { Backward } \\
\text { Recall }\end{array}$ \\
\hline $\begin{array}{c}\text { Meaningfully Similar } \\
\text { Noun Recall }\end{array}$ & .59 & .51 & .48 & .32 \\
\hline $\begin{array}{c}\text { Meaningfully Dissimilar } \\
\text { Noun Recall }\end{array}$ & .77 & .76 & .49 & .52 \\
\hline
\end{tabular}

Regarding the latter finding, examination of the proportion of correct responses (see Table 2) indicates that it arose from the absolute superiority of imagery-mnemonic Ss over sentence-memorization $S s$ in the recall of meaningfully dissimilar, as compared to meaningfully similar, nouns. Or, put another way, the relatively greater detrimental effect of meaningful similarity on Ss instructed to use an imaginal mnemonic is indicated.

When the above analysis was repeated on the scores of the half of each group scoring lowest on the selection measures, only the main effects for similar-dissimilar noun recall and backward-forward recall were significant, i.e., instructional set did not differentiate reliably.

These data support the contention that mental imagery as a mnemonic aid in recall cannot be reduced or equated to the recall of linguistic chains. Not only is the overall performance of Ss instructed to form images superior, but it is superior in the face of that group's poorer sentence memorization. In addition, the differential effect of meaningful similarity implies that some sort of difference exists in the mediational mechanisms being employed.

From the standpoint of future research on the efficacy of mental image mediation, it is important to note the very different outcomes shown in analyses of the data from the subgroups selected for high and low scores on the measures of the extent to which they were following the instructed strategies. Low-scoring Ss appear equivalent in PA recall, while high-scoring $S s$ reveal a clear superiority for image mediation. It might be argued that the two tasks used to select Ss were differentially related to the recall task such that those selected for intense image ratings were also good recallers, while those selected for good sentence memorization were not. To check on this possibility, correlations between the two selector variables and the recall scores were obtained. The correlations were .28 and .43 for the memorization and imagery groups, respectively. Though the correlation for the imagery Ss is higher, it is not reliably so. Clearly, there is no foolproof way at present to determine if instructions are being followed in the study of any private mental event. Nonetheless, these data suggest the importance of obtaining whatever circumstantial evidence is available on what and how well Ss given different instructions are actually doing. On the other hand, Bower \& Winzenz (1970) reported a study on $\mathrm{PA}$ recall, with unselected Ss showing a difference between $S$-generated sentences and images, favoring images. A number of procedural differences exist between their study and the one here reported, any one of which might account for their obtaining superiority for imagery without selection of Ss. From some preliminary observations, the writer suspects that the study times may have made the difference, i.e., 5 sec in the Bower and Winzenz study and $10 \mathrm{sec}$ in the present research. Five seconds may well have been an adequate time to generate active images but insufficient time to generate sentences. Having sentences supplied by $E$ for both groups, as opposed to S-generated mnemonics, may also have been critical. In any event, in many comparisons of learning strategies, it may be necessary to employ nonrandom assignement of $S s$ if differences among mnemonic strategies are to be detected. In fact, Paivio \& Foth (1970) indicate that the usual outcome of comparisons of imaginal and verbal mediation sets has generally shown them to be equally effective and assign this to a failure of the instructions to arouse persistent mediation strategies.

Although these data strongly suggest that visual imagery makes a mnemonic contribution to recall which cannot be equated simply to retrieving verbal chains, a question remains as to whether or not imaging is a superior mnemonic aid. Certainly, the particular instructions and S-selection procedures employed in the present experiment resulted in superior PA recall for Ss assumed to be employing an imaginal mnemonic. Bower (1969) argues that superiority of an imaginal over a linguistic mnemonic, when it is shown, may be due to the mental-imagey-instructed
Ss' retrieving from both a pictorial and a verbal memory store, while sentence-reading, -generating, or -memorizing Ss are retrieving only from a verbal store. It is not clear to the writer why instructions to irnage result in incidental verbal storage, while instructions to verbalize do not lead to incidental storage of images. Certainly, the absence of a relationship between the degree of incidental sentence memorization and $P A$ recall by mental-imagery-instructed $S$ s in the present study does not lend support to the hypothesis. However, the limited sample size for the correlation $(\mathrm{N}=11)$ makes relating this to the Bower hypothesis only suggestive.

It might be argued that the superiority of imagery was due to the fact that the tasks designed to measure individual differences in the degree to which the instructions were being followed were differentially reinforcing to the recall of the paired associates. That is, sentence correction may have been a distraction and image elaboration an aid to the primary task of $P A$ recall. What is needed are agreed-upon indices of the predisposition and proficiency of Ss toward particular mnemonic strategies being studied so that $S s$ can be preexperimentally assigned to various conditions.

These data lend strong support to the belief that mental imagery is a useful construct in the theoretical analysis of memory and of potential practical import to those concerned with the technology of education.

BOWER, G. H. Mental imagery and associative learning. Fifth annual symposium on cognition. Carnegie-Mellon University, Pittsburgh, Pa., 1969.

BOWER, G. H., \& WINZENZ, D. Comparison of associative learning strategies. Psychonomic Science, 1970, 20, 119-120.

BUGELSKI, B. R. Words and things and images. American Psychologist, 1970, 25, $1002-1012$.

PAIVIO, A. Mental imagery in associative learning and memory. Psychological Review, 1969, 76, 241-263.

PAIVIO, A., \& FOTH, D. Imaginal and verbal mediators and noun concreteness in paired-associate learning: The elusive interaction. Journal of Verbal Learning \& Verbal Behavior, 1970, 9, 384-390.

PAIVIO, A., \& YUILLE, J. Changes in associative strategies and paired-associate learning over trials as a function of word imagery and type of learning set. Journal of Experimental Psychology, 1969, 79 458-463.

PAIVIO, A., YUILLE, J., \& MADIGAN, Concreteness, imagery, and meaningfulness values for 925 nouns. Journal of Experimental Psychology Monograph Supplements, 1968, 76(1, Part 2), 1-25.

RIMM, D. C., ALEXANDER, R. A., \& EILES, R. R. Effects of different mediational instructions and sex of subject on paired-associate leaming of concrete nouns. Psychological Reports, 1969, 25, 935-940. 